\title{
Swallowed button batteries: is there a consensus on management?
}

\author{
J G N Studley, I P Linehan, A L Ogilvie, B L Dowling
}

\begin{abstract}
The optimum management of ingested button batteries was ascertained by postal questionnaire sent to 608 members of the endoscopic and paediatric sections of the British Society of Gastroenterology. Some 312 returns were suitable for analysis: $36 \cdot 2 \%$ of the respondents were not concerned about ingested button batteries and gave no treatment, $6.4 \%$ used medical treatment, $48.4 \%$ removed them under certain circumstances, and $9 \%$ did not know how to manage the problem. Emetics and $\mathrm{H}_{2}$ antagonists or antacids were often used for batteries in the oesophagus, stomach, and duodenum and laxatives were commonly prescribed for batteries in the small and large bowel. Of the $48.4 \%$ who felt batteries should be removed under certain circumstances, $78 \%$, $72 \%$, and $48 \%$ extracted them from the oesophagus, stomach, and duodenum respectively within 24 hours of ingestion. The main reason for operative removal from the small and large bowel was failure of the battery to progress. Current management is therefore variable. Heavy metal poisoning may be occurring more frequently than is suggested in the published reports.
\end{abstract}

Button batteries are small disc-shaped power units used in various devices including cameras, digital watches, hearing aids, and calculators. Ingestion of these batteries by children is not an infrequent occurrence. Most batteries pass through the gastrointestinal tract without adverse effect, ${ }^{1}$ but there have been reports of important clinical problems.

The batteries currently available consist of various substances including a heavy metal salt and an alkali. Mercuric oxide, silver oxide, manganese dioxide, zinc oxide, or lithium hydroxide are among the metallic salts commonly present. ${ }^{1-4}$ The alkali is usually sodium or potassium hydroxide. ${ }^{156}$ Adverse effects may occur as a result of local damage or heavy metal poisoning.

Local damage in the gastrointestinal tract is caused by a combination of pressure necrosis, direct corrosive action, and low voltage burn. ${ }^{17}$ Corrosion of the battery leads to increasing leakage of the constituents ${ }^{89}$ and eventually rupture of the case with release of both alkali and inorganic constituents into the gastrointestinal tract.

This daunting potential problem must be put into perspective. Major corrosive problems have almost always been due to batteries lodged in the oesophagus. Two children have died as a consequence of local corrosive effects in this region. In one, a tracheo-oesophageal fistula developed ${ }^{5}$ and in the other the aortic arch was corroded leading to massive haemorrhage. ${ }^{10}$ Three further tracheo-oesophageal fistulae have been reported in children, all of whom survived. ${ }^{6112}$ In one child an oesophageal stricture developed ${ }^{+}$and another suffered a leak from the oesophagus, which resolved. ${ }^{1}$ Once beyond the oesophagus the only reported corrosive problem has been a battery that lodged in a Meckel's diverticulum, causing it to perforate. ${ }^{13}$ Most ingested batteries pass through the gastrointestinal tract without causing symptoms. Symptoms that have been recorded, however, include anorexia, nausea, vomiting, and the passage of dark grey or black stools. ${ }^{814}$ is 16 Batteries may also cause mucosal erosions, gastritis, and minor upper gastrointestinal bleeding. ${ }^{161117}$

Heavy metal poisoning is possible after disintegration of the battery casing, and if it occurs it has always been associated with mercury containing cells. ${ }^{21718}$ Toxic serum concentrations of mercury may occur and the patient may require chelation treatment. ${ }^{21718}$

As a result of these potential problems several authors have recommended an aggressive approach to ensure removal of swallowed button batteries. ${ }^{6815}$ More recent evidence indicates, however, that most button batteries pass through the gastrointestinal tract with no adverse effects, and because of this a more conservative approach has been recommended. ${ }^{1}{ }^{319}$ With this conflict in views we felt it would be helpful to see if there was a consensus of opinion regarding management.

\section{Methods}

A questionnaire relating to management of ingested button batteries was sent to members of the endoscopic and paediatric sections of the British Society of Gastroenterology.

\section{Results}

Some 608 questionnaires were sent and 333 $(54 \cdot 8 \%)$ were returned. Unfortunately, 21 of the returns were not filled in adequately or had been sent to non-clinicians. These were removed from the analysis leaving 312 questionnaires for assessment. The respondents were divided into four main groups:

Group I. One hundred and thirteen $(36 \cdot 2 \%)$ were not concerned about button batteries and gave no treatment.

Group II. Twenty (6.4\%) indicated that they used various forms of medical treatment, but did not attempt removal of the battery.

Group III. One hundred and fifty one (48.4\%) elected to remove the battery under certain circumstances. 
TABLE I Group II -drug treatment given by 20 respondents who did not advocate removal of batteries

\begin{tabular}{lcclcc}
\hline Drug treatment & Oesophagus & Stomach & Duodenum & $\begin{array}{c}\text { Small } \\
\text { bowel }\end{array}$ & $\begin{array}{c}\text { Large } \\
\text { bowel }\end{array}$ \\
\hline No treatment & 5 & 4 & 7 & 6 & 5 \\
Emetics & 12 & 10 & 3 & -5 & -1 \\
Antacids or $\mathrm{H}_{2}$ antagonists & 3 & 9 & 6 & 13 & 15 \\
Laxatives & - & - & 7 & \\
\hline
\end{tabular}

TABLE II Group II - treatment given by 151 respondents who remove batteries in certain circumstances

\begin{tabular}{lccccc}
\hline & Oesophagus & Stomach & Duodenum & $\begin{array}{c}\text { Small } \\
\text { bowel }\end{array}$ & $\begin{array}{c}\text { Large } \\
\text { bowel }\end{array}$ \\
\hline $\begin{array}{l}\text { No treatment } \\
\text { Medical treatment }\end{array}$ & 1 & 3 & 12 & 16 & 23 \\
Remove: & 2 & 8 & 20 & 49 & 58 \\
$\quad$ Immediately & 90 & 71 & 50 & 10 & 6 \\
Within 24 hrs & 28 & 37 & 21 & 13 & 7 \\
If symptoms & 4 & 6 & 7 & 15 & 8 \\
If no progress & 1 & 7 & 17 & 41 & 38 \\
$\rightarrow$ 8 hrs & 1 & 1 & 1 & 5 & 5 \\
$\rightarrow 24$ hrs & - & 2 & 8 & 12 & 11 \\
$\rightarrow 48$ hrs & - & 1 & 2 & 6 & 4 \\
$\rightarrow 72$ hrs & - & 1 & 1 & 4 & 3 \\
$\rightarrow 14$ days & - & 1 & 1 & 1 & 2 \\
\hline
\end{tabular}

$\star$ Numbers below indicate times waited if the battery has not progressed

TABLE III Group III reasons given by 79 respondents for removal of batteries which have passed the pylorus

\begin{tabular}{lr}
\hline Failure to progress: & \\
Unspecified time & 41 \\
$\rightarrow 24 \mathrm{hrs}$ & 10 \\
$\rightarrow 48 \mathrm{hrs}$ & 4 \\
$\rightarrow 72 \mathrm{hrs}$ & 3 \\
$\rightarrow 7$ days & 1 \\
Disruption & 9 \\
Symptoms & 7 \\
Perforation & 2 \\
Meckel's diverticulum & 2 \\
Small bowel & 1 \\
Lithium battery & 1 \\
Mercury battery & 1 \\
Blood levels & 1 \\
Childhood & 1 \\
Risk to survival & 1 \\
\hline
\end{tabular}

\section{GROUP III - REMOVAL OF BATTERIES}

One hundred and fifty one respondents removed the battery from the gastrointestinal tract under certain circumstances. The details are shown in Table II.

Several further questions were then asked of clinicians in this category but not all respondents gave answers to each section.

(1) How do you attempt to remove them initially if in the oesophagus or stomach? (127 answers). One hundred and twenty favoured the fibreoptic endoscope, seven the rigid oesophagoscope, while none resorted to surgery.

(2) If endoscopic methods fail do you resort to surgery? (98 answers). Seventy nine respondents indicated that they would resort to surgery, although two did specify that this would occur only if the battery was retained in the oesophagus. Nineteen would not proceed to surgery.

(3) Would you ever operate to remove a battery that

TABLE IV Group III - drug treatment given by doctors who advocated removal in certain circumstances

\begin{tabular}{lllllc}
\hline Drug treatment & Oesophagus & Stomach & Duodenum & $\begin{array}{c}\text { Small } \\
\text { bowel }\end{array}$ & $\begin{array}{c}\text { Large } \\
\text { bowel }\end{array}$ \\
\hline No treatment & 47 & 40 & 36 & 25 & 20 \\
Emetics & 5 & 4 & - & - & - \\
Antacids or $\mathrm{H}_{2}$ antagonists & 9 & 17 & 14 & 2 & 72 \\
Laxatives & - & 4 & 21 & 64 & 72 \\
Metoclopramide & - & 1 & 1 & 2 & - \\
\hline
\end{tabular}

has gone beyond the pylorus? (111 answers). Seventy nine would operate under these circumstances, while 32 would not. For the 79 who would resort to surgery, the details for removal are shown in Table III.

(4) What would your medical management be with a battery in the oesophagus, stomach, duodenum, small bowel, or large bowel? The answers to this question are shown in Table IV.

(5) Have you ever seen a patient with heavy metal poisoning caused by a button battery? Seven (4.6\%) indicated that they had. One clinician specified a patient with renal failure, while the others did not give details.

\section{Discussion}

It should be emphasised that this survey reflects current attitudes to the management of swallowed button batteries, and does not indicate optimum management. There is a wide variety of approaches $-36 \%$ of the respondents were unconcerned about button battery ingestion while $48 \%$ elected to remove them under some circumstances.

In Group II, clinicians who use medical treatment only, most respondents use emetics for batteries that are in the oesophagus and stomach. There is little evidence in the published reports that this particular approach has been successful. ${ }^{1616}$ In addition, the point has been made that emetics may be contraindicated because of the possibility of the battery creating airway obstruction..$^{13}$ If the battery is in the stomach, $\mathrm{H}_{2}$ antagonists or antacids are often used. The rationale of this treatment is that reduction of gastric acidity should, theoretically, minimise corrosion of the battery. ${ }^{1}$ Nolan and Tucker ${ }^{9}$ have observed increased corrosion of mercury batteries in an acid environment. Experiments have shown that cimetidine had no protective effect with batteries in the stomach of dogs although certain antacids did seem to reduce corrosion..$^{20}$ From another point of view, should a battery leak and alkali be released, the caustic effect would be reduced by gastric acidity, which might possibly reduce damage. ${ }^{6}$ Thus the case for using $\mathrm{H}_{2}$ antagonists or antacid is uncertain.

The answers of those who felt active intervention was indicated were examined (Group III). It was clear that most respondents felt that the battery should be removed from the oesophagus. Sixty per cent removed the battery immediately and a further $18 \%$ of clinicians removed it within 24 hours. Because the two deaths and all but one of the cases of severe morbidity associated with corrosion have occurred with the battery in the oesophagus, removal would be strongly supported. But is it safe to wait for a period of time before removing the battery? In the patients with severe oesophageal damage previously mentioned, the battery had been present for six hours, ${ }^{1}$ in excess of 22 hours, ${ }^{12} 24$ hours, ${ }^{5} 4.5$ days, ${ }^{10}$ five days, ${ }^{6}$ and two ${ }^{11}$ and four weeks. ${ }^{4}$ Experimental evidence has shown that mucosal necrosis develops within one hour and ulceration in two hours if a battery is present in one position in the oesophagus, gastric antrum, or mid or distal ileum in dogs - after seven hours there was no evidence of perforation. Maves $e t$ a $l^{12}$ have 
shown that in the oesophagus of cats perforation occurs in eight to 12 hours. Clinical evidence shows therefore that perforation has occurred as early as six hours and experimental evidence indicates that this may occur from eight hours onwards. In view of this, it seems that urgent removal of a battery in the oesophagus is the correct approach.

With a battery identified in the stomach, $47 \%$ of the respondents removed it as soon as possible and a further $25 \%$ within 24 hours. No serious case of morbidity has been reported with a battery in this position, apart from separation of the battery after two days, which led to toxic serum mercury concentrations. ${ }^{17}$ Superficial erosions have been noted as early as two hours after ingestion and minor bleeding after 18 hours. ${ }^{16}$ Experimental evidence in dogs has shown ulceration within two hours with no evidence of perforation by seven hours. ${ }^{6}$ Thus, there is much less evidence to support removing the battery from the stomach than the oesophagus.

Forty eight per cent of the respondents in this group removed the battery from the duodenum within 24 hours. Once it had passed beyond this position, however, only a minority felt removal was necessary unless there was no progress over varying amounts of time, most indicating that they removed a battery within two days. This is supported by the patient with the perforated Meckel's diverticulum in whom the battery had been in the gastrointestinal tract for three days. ${ }^{13}$ The main question here is: how long is it safe to wait? Experimental evidence suggests that no serious damage is caused by leaving a battery in one place in the small bowel for seven hours, ${ }^{6}$ while the perforation that occurred in clinical practice was after three days. ${ }^{13}$ The optimal timing is therefore unclear.

Laxatives were prescribed by $42 \%$ of the respondents for batteries identified in the small bowel and by $48 \%$ once batteries had reached the large bowel. This approach has also been advocated in the published reports. ${ }^{16}$

When we consider the method by which the battery is removed from the upper gastrointestinal tract, it is not surprising that most doctors favoured the fibreoptic endoscope. Magnetic removal with fluoroscopy was not used. ${ }^{21}{ }^{22}$ No one resorted to surgery in the first instance. Asked whether they would proceed to surgery if endoscopy failed, 79 of the respondents said they would (two specifying that they would use surgery only if the battery were in the oesophagus) and 19 stated they would not. The latter figure may well indicate a natural reluctance to perform surgery in view of the low incidence of problems that may occur.

Another area of contention is the question of how long it is safe to leave a battery in the gastrointestinal tract without the danger of rupture. This question is impossible to answer because the state of the battery when it was swallowed can be extremely variable. Corrosion is less in discharged batteries. In vitro studies have shown a direct relation between corrosion of cells in an acid environment and the discharge status of the battery. ${ }^{20}$ Batteries have been noted to rupture between 36 and 48 hours after ingestion. ${ }^{281+17}$

What is the danger of mercury poisoning once the battery has separated? There have been three reports of raised mercury concentrations, after a battery separated in the stomach ${ }^{17}$ and colon. ${ }^{218}$ It is well documented that batteries can rupture without causing mercury poisoning ${ }^{81+}$ and this is supported by reports stating that the risk of poisoning is low. ${ }^{16}$ This may be partly due to the conversion of mercuric oxide to metallic mercury, which is not absorbed in the bowel. ${ }^{123}$ The magnitude of the danger of mercury poisoning is therefore unclear but the published evidence indicates that it is low.

One of the most astonishing features of the survey was that seven respondents had seen patients with heavy metal poisoning caused by swallowing button batteries. This number is certainly not reflected in the published reports. Respondents were not asked to give further details of this particular aspect, nevertheless, it would seem that heavy metal poisoning may be occurring more often than is evident from published reports.

At Northampton we have developed a relatively aggressive policy towards the management of patients who have swallowed these batteries. In most patients the exact nature of the battery, its constituents, the remaining power, and whether it was corroded or damaged cannot be determined. The latter point is important because used batteries are reported to cause less alkaline change than new cells. ${ }^{6}$ Consequently, we assume all to be mercury batteries with full function unless we can prove otherwise. If the battery is in the oesophagus we remove it with a flexible endoscope as soon as possible. If the battery is in the stomach, we are prepared to wait for 24 hours after ingestion to see if it will pass beyond the pylorus. Metoclopramide is prescribed in order to enhance gastric emptying but we do not give antacids or $\mathrm{H}_{2}$ antagonists. If the battery is still in the stomach 24 hours later, removal is attempted with a flexible endoscope. If this is not successful a laparotomy will be performed. In patients in whom the battery has passed beyond the pylorus, laxatives will be prescribed. The abdomen is then $x$ rayed every 24 hours to ensure progress is being made. If the battery is held in any one position for 24 hours or more or if the patient develops symptoms, it is removed surgically. If the battery ruptures, it is removed, mercury concentrations in the serum and urine are monitored, and chelating agents are given if necessary.

In the past 18 months five children who have swallowed a button battery have been referred to us. All initial $x$ rays showed the battery to be in the stomach. At that time, it was our policy to remove batteries from the oesophagus and stomach once suitable arrangements had been made. In the first patient endoscopy showed that the battery had passed out of the stomach. Subsequently, we have insisted that $x$ rays are taken when patients are on the way to theatre. In one patient this check $x$ ray showed that the battery had passed on, and no intervention was indicated apart from laxatives. In the remaining three patients the batteries were still in the 
stomach on check $x$ ray. Two of these batteries were removed using the flexible endoscope with a Dormia basket. We could not remove the battery in the fifth patient because it had adhered to the fundus of the stomach. No further procedure was undertaken at that time, but 12 hours later the patient had another $x$ ray and the battery had not moved. Another attempt at flexible endoscopic removal was made, but again this was unsuccessful. Laparatomy and gastrotomy were performed and the battery was removed. It separated almost immediately, releasing its contents.

In summary, this survey shows that there is wide variation in the management of swallowed button batteries. Undoubtedly, most of these batteries pass through the gastrointestinal tract with no adverse effects. Reports of morbidity and mortality associated with the batteries are few, but have led many doctors to a more aggressive approach. Heavy metal poisoning after button battery ingestion may occur more frequently than is evident in the published reports at present.

We would like to acknowledge the help of Ann Skinner who assisted us in obtaining the relevant publications.

1 Litovitz TL. Button battery ingestions. A review of 56 cases. FAMA 1983; 249: 2495-500

2 Kulig K, Rumack CM, Rumack BH, Duffy JP. Disk battery ingestion. Elevated urine mercury levels and enema removal of battery fragments. FAMA 1983; 249: 2502-4.
3 David TJ, Ferguson AP. Management of children who have swallowed button batteries. Arch Dis Child 1986; 61: 321-2.

4 Kost KM, Shapiro RS. Button battery ingestion: a case report and review of the literature. $\mathcal{F}$ Otolaryngol 1987; 16: 252-7.

5 Blatnik DS, Toohill RJ, Lehman RH. Fatal complication from an alkaline battery foreign body in the esophagus. Ann Otol an alkaline battery

6 Votteler TP, Nash JC, Rutledge JC. The hazard of ingested alkaline disk batteries in children. $\mathcal{F} A M A$ 1983; 249: alkaline

7 Katz L, Cooper MT. Danger of small children swallowing hearing aid batteries. F Otolaryngol 1978; 7: 467.

8 Temple DM, McNeese MC. Hazards of battery ingestion. Pediatrics 1983; 71: 100-3

9 Nolan M, Tucker I. Health risks following ingestion of mercury and zinc air batteries. Scand Audiol 1981; 10:

10 Shabino CL, Feinberg AN. Esophageal perforation secondary to alkaline battery ingestion. $\mathcal{f} A C E P$ 1979; 8: 360-2

11 Janik JS, Burrington JD, Wayne ER, Foley LC. Alkaline battery ingestion. Colo Med 1982; 79: 404-5.

12 Maves MD, Carithers JS, Birck HG. Esophageal burns Maves MD, Carithers JS, Birck HG. Esophageal burns
secondary to disc battery ingestion. Ann Otol Rhinol Secondary to disc battery

13 Willis GA, Ho WC. Perforation of Meckel's diverticulum by an alkaline hearing aid battery. Can Med Assoc $\mathcal{F} 1982 ; 126$ : 497-8.

14 Barros D'Sa EA, Barros D'Sa AAB. Mercury battery ingestion. BrMed F 1979; i: 1218.

15 Reilly DT. Mercury battery ingestion. Br Med f 1979; i: 859.

16 Litovitz TL. Battery ingestions: Product accessibility and clinical course. Pediatrics 1985; 75: 469-76.

17 Mant TGK, Lewis JL, Mattoo TK, et al. Mercury poisoning after disc-battery ingestion. Hum Toxicol 1987; 6: 179-81.

18 Mofenson HC, Greensher J, Caraccio TR, Danoff R. Ingestion of small flat disc batteries. Ann Emerg Med 1983; 12: 88-90.

19 Kiely B, Gill D. Ingestion of button batteries: hazards and management. BrMed F 1986; 293: 308-9.

20 Litovitz T, Butterfield AB, Holloway RR, Marion LI. Button battery ingestion: assessment of therapeutic modalities and battery discharge state. $\mathcal{F}$ Pediatr 1984; 105: 868-73.

21 Jaffe RB, Corneli HM. Fluoroscopic removal of ingested alkaline batteries. Radiology 1984; 150: 585-6.

22 Ito Y, Ihara N, Sohma S. Magnetic removal of alkaline batteries from the stomach. $\mathcal{F}$ Pediatr Surg 1985; 20: 250-1.

23 Barber TE, Menke RD. The relationship of ingested iron to the absorption of mercuric oxide. Am $\mathcal{F}$ Emerg Med 1984; 2: $500-3$. 